\title{
EROS 2 photometry of probable R Coronae Borealis stars in the Small Magellanic Cloud ${ }^{\star}$
}

\author{
P. Tisserand ${ }^{1}$, J. B. Marquette ${ }^{2}$, J. P. Beaulieu ${ }^{2}$, P. de Laverny ${ }^{3}$, É. Lesquoy ${ }^{1,2}$, A. Milsztajn ${ }^{1}$, C. Afonso ${ }^{1,4}$, \\ J. N. Albert ${ }^{5}$, J. Andersen ${ }^{6}$, R. Ansari ${ }^{5}$, É. Aubourg ${ }^{1}$, P. Bareyre ${ }^{1,4}$, F. Bauer ${ }^{1}$, G. Blanc ${ }^{1,7,8}$, X. Charlot $^{1}$, \\ C. Coutures ${ }^{1}$, F. Derue ${ }^{5,9}$, R. Ferlet ${ }^{2}$, P. Fouqué ${ }^{10,11}$, J. F. Glicenstein ${ }^{1}$, B. Goldman ${ }^{1,4}$, A. Gould ${ }^{4,12}$, \\ D. Graff ${ }^{13}$, M. Gros ${ }^{1}$, J. Haissinski ${ }^{5}$, C. Hamadache ${ }^{1}$, J. de Kat ${ }^{1}$, T. Lasserre ${ }^{1}$, L. Le Guillou ${ }^{1}$,

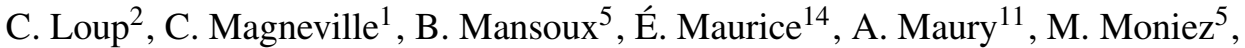 \\ N. Palanque-Delabrouille ${ }^{1}$, O. Perdereau ${ }^{5}$, L. Prévot ${ }^{14}$, Y. Rahal $^{5}$, N. Regnault ${ }^{5}$, \\ J. Rich $^{1}$, M. Spiro ${ }^{1}$, A. Vidal-Madjar ${ }^{2}$, L. Vigroux ${ }^{1}$, and S. Zylberajch ${ }^{1}$ \\ 1 CEA, DSM, DAPNIA, Centre d'Études de Saclay, 91191 Gif-sur-Yvette Cedex, France \\ 2 Institut d'Astrophysique de Paris, INSU CNRS, 98bis boulevard Arago, 75014 Paris, France \\ e-mail: marquett@iap.fr \\ 3 Cassiopée UMR 6202, Observatoire de la Côte d'Azur, BP 4229, 06304 Nice Cedex 4, France \\ 4 Collège de France, Physique Corpusculaire et Cosmologie, IN2P3 CNRS, 11 place Marcelin Berthelot, \\ 75231 Paris Cedex 05, France \\ ${ }^{5}$ Laboratoire de l'Accélérateur Linéaire, IN2P3 CNRS, Université de Paris-Sud, 91405 Orsay Cedex, France \\ 6 Astronomical Observatory, Copenhagen University, Juliane Maries Vej 30, 2100 Copenhagen, Denmark \\ 7 Osservatorio Astronomico di Padova - INAF, vicolo dell'Osservatorio 5, 35122 Padova, Italy \\ ${ }^{8}$ Université Paris 7 Denis Diderot, 2 place Jussieu, 75005 Paris, France \\ 9 LPNHE, IN2P3 CNRS and Universités Paris 6 and Paris 7, 4 place Jussieu, 75252 Paris Cedex 05, France \\ 10 Observatoire Midi-Pyrénées, UMR 5572, 14 avenue Édouard Belin, 31400 Toulouse, France \\ 11 European Southern Observatory, Casilla 19001, Santiago 19, Chile \\ 12 Department of Astronomy, Ohio State University, Columbus, OH 43210, USA \\ 13 Department of Math and Science, US Merchant Marine Academy, Kings Point, NY 11024, USA \\ 14 Observatoire de Marseille, 2 place Le Verrier, 13248 Marseille Cedex 04, France
}

Received 4 March 2004 / Accepted 26 May 2004

\begin{abstract}
EROS 2 (Expérience de Recherche d'Objets Sombres) conducted a survey of the SMC between July 1996 and February 2003 in two EROS broad-band colours, $V_{\mathrm{E}}$ and $R_{\mathrm{E}}$. The photometric data of 4.2 million stars have been searched for behaviour typical of R Coronae Borealis (RCB) candidates such as drastic changes in magnitude. Five objects have been found, four of them being catalogued in the Simbad database as RAW 21, RAW 233, RAW 476, and [MH95] 431 with confirmed carbon-rich atmospheres, characteristic of RCB. From the EROS 2 light curve of RAW 21 and its spectrum reported by Morgan et al. (2003), we confirm that it is the first RCB to be found in the SMC. The other objects are new RCB candidates with absolute luminosity and colour close to those found for RCBs in the LMC. We propose that 2 of them are DY Per-like RCBs.
\end{abstract}

Key words. stars: carbon - galaxies: Magellanic Clouds

\section{Introduction}

As described by Clayton (1996) in a detailed review, R Coronae Borealis (RCB) stars are carbon-rich supergiants in a phase of rapid evolution. They are born-again giants for which two evolutionary scenarios have been proposed: either a merger of two white dwarfs or a final He-shell flash in a post-AGB star.

^ Based on observations made with the CNRS/INSU MARLY telescope at the European Southern Observatory, La Silla, Chile.
They exhibit extreme and irregular changes in brightness, up to $8 \mathrm{mag}$ in visible light, caused by the obscuration of the stellar surface by newly formed dust clouds. They are rare objects: about 50 are known in the Galaxy although population estimates are of a few thousand. Furthermore, no Galactic RCBs have accurate distances so that the absolute luminosities of these variables are poorly known. This is why it is important to detect RCB objects in external galaxies, such as the Magellanic Clouds (MC), whose distances are known. Studying RCBs in 
such low-metallicity systems is also crucial in order to better understand their evolutionary status and the time spent in the RCB phase. Thanks to the work of the MACHO collaboration on the LMC (Alcock et al. 1996, 2001), the number of detected RCB variables in this galaxy has increased from 3 to 17 ; they estimate that the total number of LMC RCBs is less than 100. However, Alcock et al. (2001) stated in the same study that none were found in the search performed in their SMC fields.

More recently, Morgan et al. (2003) reported a spectroscopic study of a sample of $\sim 2300 \mathrm{MC}$ carbon stars from which it appeared that six of them show $\mathrm{C}_{2}$ bands but weak or absent $\mathrm{CN}$ bands, a typical spectral signature of RCBs. They claimed that one of them is the first RCB candidate detected in the SMC, namely RAW 21 . This acronym RAW comes from the catalog of carbon stars by Rebeirot et al. (1993) who spectrophotometrically identified 1707 objects in the SMC.

The present paper reports on the mining of the EROS 2 (Expérience de Recherche d'Objets Sombres) database in a search for RCB candidates in the SMC. Results of this study are available at the URL http://eros.in2p3.fr/ Variables/RCB/RCB-SMC.html.

\section{Observational data}

The EROS 2 project made use of a 1-m telescope located at La Silla Observatory, Chile, to extensively search for the baryonic dark matter of the Galactic halo. The method was based on the microlensing effect as described by Afonso et al. (2003), Derue et al. (2001), Lasserre et al. (2000), and Palanque-Delabrouille et al. (1998). The observations were performed between July 1996 and February 2003 with two wide field cameras $\left(0.7^{\circ}\right.$ in right ascension $\times 1.4^{\circ}$ in declination) behind a dichroic cube splitting the light beam into two broad passbands. The so-called "blue" channel (420-720 nm, hereafter magnitudes $V_{\mathrm{E}}$ ) overlapped the $V$ and $R$ standard bands while the "red" one (620-920 nm, hereafter magnitudes $R_{\mathrm{E}}$ ) roughly matched the $I$ standard band. Each camera was a mosaic of eight $2048 \times 2048$ CCDs with a pixel size of $0.6^{\prime \prime}$ on the sky.

For the observations, the SMC was separated into ten fields, each of them having a surface of $\sim 1 \mathrm{deg}^{2}$. These fields were called sm001 to sm010 and labelling of the stars was identical to that done by Derue et al. (2002). The photometry of individual images and the reconstruction of the light curves were processed using the Peida package which has been specifically developped for the EROS experiment (Ansari 1996). An estimation of the photometric accuracy is discussed by Derue et al. (2002). Recently all available SMC data were used to recompute the entire sample of light curves. Over 5.6 million sources were detected on our template images. These images are constructed by co-adding the 15 best seeing images of each field obtained between January 1997 and May 2001. Once artifacts and the faintest objects were eliminated, we analysed more than 4.2 million light curves. Stars were imaged on 650 epochs for outer fields and 950 epochs for inner fields.

\section{Mining the EROS database}

As stated by Clayton (1996) RCB light curves in the optical exhibit the following properties: initial decline of 3 to 6 mag in about 50-100 days (at irregular intervals); fast or slow recoveries and/or multiple declines follow; final recovery may be slow. Guided by these properties, we defined criteria to search for a few RCB candidates among millions of objects. First we extracted the most variable stars by correlating light curves taken through the "red" channel and the "blue" channel: objects with a correlation coefficient greater than 0.5 were kept (about $5 \%$ of the stars). After this step a medium flux was calculated in each colour as $F_{\text {med }}=1 / 2 \times\left(F_{\max }+F_{\min }\right)$ where $F_{\max }$ and $F_{\min }$ are the maximum and minimum fluxes, respectively. The quantities $F_{\max }$ and $F_{\min }$ were determined as the averages of 10 points at maximum and at minimum, respectively, chosen after elimination of the 5 most extreme points in both maximum and minimum domains. The number of times a light curve crosses $F_{\text {med }}$ by at least 3 consecutive points in either "red" or "blue" colour was used to select stars for which this quantity was lower than 12 . Remaining objects were then kept if $2.5 \times \log _{10}\left(F_{\max } / F_{\min }\right)>2.0 \mathrm{mag}$ in either "red" or "blue" colour. This yielded a sample of 429 stars which were visually inspected to eliminate Miras or nova-like light curves and photometric artifacts, while still retaining stars for which a rapid decrease (or increase) of luminosity is observed.

\section{Detection efficiency}

There was until now no confirmed RCB star in the SMC. We have thus estimated our RCB detection efficiency by applying the cuts described in the previous section to the 17 known LMC RCB stars, as listed in Table 1 of Alcock et al. (2001). One of these 17 stars (HV 12842) is outside our fields and is not considered further. Using the light curves measured by Alcock et al. (2001) between mid-1992 and the end of 1999, we find that 14 stars out of 16 satisfy our selection criteria. Among the 2 rejected stars, one is a DY Per.

In addition, we have searched our LMC database for these 16 stars light curves and we have checked whether they satisfy the selection criteria, based on EROS 2 data alone (July 1996 to February 2003). Seven of them are selected, none of them being a DY Per. None of the remaining 9 stars from Alcock et al. (2001) showed large enough variations satisfying our selection criteria during the EROS 2 observing period. This allows a rough estimate of the probability to detect an RCB candidate with about 450 photometric measurements over 6.5 years at about $50 \%$. (This number should be typical for the LMC; in the SMC, where we have 650 to 950 epochs, our detection efficiency might be higher.) We intend to report on these LMC stars in a future publication.

\section{The EROS2 SMC RCB candidates}

After the visual inspection of the 429 selected light curves, five objects remain which are listed in Table 1. Four of these candidates were retrieved in the Simbad database. The abbreviation [MH95] in Table 1 refers to the spectroscopic survey 
Table 1. Coordinates of the EROS 2 SMC RCB candidates.

\begin{tabular}{llc}
\hline \hline Simbad identifier & EROS2-RCB identifier & J2000 coordinates \\
\hline RAW 21 & J003747-733902(sm0102120592b) & $00: 37: 47.07-73: 39: 02.1$ \\
RAW 233 & J004407-724416(sm0077k11497b) ${ }^{a}$ & $00: 44: 07.45-72: 44: 16.3$ \\
RAW 476 & J004822-734104(sm0014k11612b) & $00: 48: 22.87-73: 41: 04.7$ \\
[MH95] 431 & J004014-741121(sm0106m19412b) & $00: 40: 14.65-74: 11: 21.2$ \\
$n / a$ & J005718-724235(sm0067m28134b) ${ }^{b}$ & $00: 57: 18.12-72: 42: 35.3$ \\
\hline$a$ MACHO identifier: 208.15571 .60$. &
\end{tabular}

${ }^{a}$ MACHO identifier: 208.15571.60.

${ }^{b}$ MACHO identifier: 207.16426.1662; hereafter identified as sm0067m28134b.

of 1185 carbon stars in the SMC by Morgan \& Hatzidimitriou (1995). The J2000 coordinates of the objects are those measured on the EROS 2 template images with an average accuracy of $1^{\prime \prime}$.

Figures 1 to 5 display the light curves of the RCB candidates in both $V_{\mathrm{E}}$ and $R_{\mathrm{E}}$ colours together with the temporal evolution of the colour index $V_{\mathrm{E}}-R_{\mathrm{E}}$. Also indicated by vertical lines are the epochs of 2MASS and (mostly unpublished) DENIS observations. Data for RAW 233 and sm0067m28134b are available from the Lightcurve Retrieval on the MACHO webserver $^{1}$ for the coordinates given in Table 1 . Some CCDs of the EROS "red" camera had technical problems from time to time. This is the reason for the wide gaps in the $R_{\mathrm{E}}$ data, which unfortunately coincide for some objects with interesting magnitude changes detected with the "blue" camera. However it remains clear that for all objects the light and colour variations are large and erratic. For the sake of clarity, error bars have been omitted on the colour index in Figs. 1 to 5, and points for which the error on either $V_{\mathrm{E}}$ or $R_{\mathrm{E}}$ is greater than 1 mag have been removed.

Figure 6 shows the colour-magnitude excursion of each object. The solid lines indicate the direction of a reddening vector corresponding to a standard extinction, which is probably a rough approximation for a carbon-rich atmosphere. For the EROS passbands it is determined according to Eqs. (1) and (3) of Cardelli et al. (1989) with effective wavelengths equal to $0.626 \mu \mathrm{m}$ and $0.788 \mu \mathrm{m}$ for $V_{\mathrm{E}}$ and $R_{\mathrm{E}}$, respectively. The quantities $A_{\mathrm{V}}$ and $E(B-V)$ for the SMC are taken from the NASA/IPAC Extragalactic Database (NED).

Figure 7 shows the positions of the RCB candidates in a near infrared two-colour diagram constructed with the 2MASS data obtained through VizieR for the carbon stars of the catalog by Morgan \& Hatzidimitriou (1995). The solid line corresponds to a model by Westerlund et al. (1991) giving the colour of the SMC carbon stars. It should be noticed that the colours of RAW 476, sm0067m28134b and RAW 21 seem to be linearly correlated as are the LMC RCBs of Alcock et al. (2001). The locations of these stars are also compared to the locus of a combination of two blackbodies, e.g. a $5500 \mathrm{~K}$ star and a $1000 \mathrm{~K}$ dust shell in various proportions ranging from all star (lower end of the dashed line) to all shell (upper end of the dashed line), as proposed by Feast (1997).

\footnotetext{
${ }^{1}$ http://store.anu.edu.au: 3001//cgi-bin/lc.pl
}

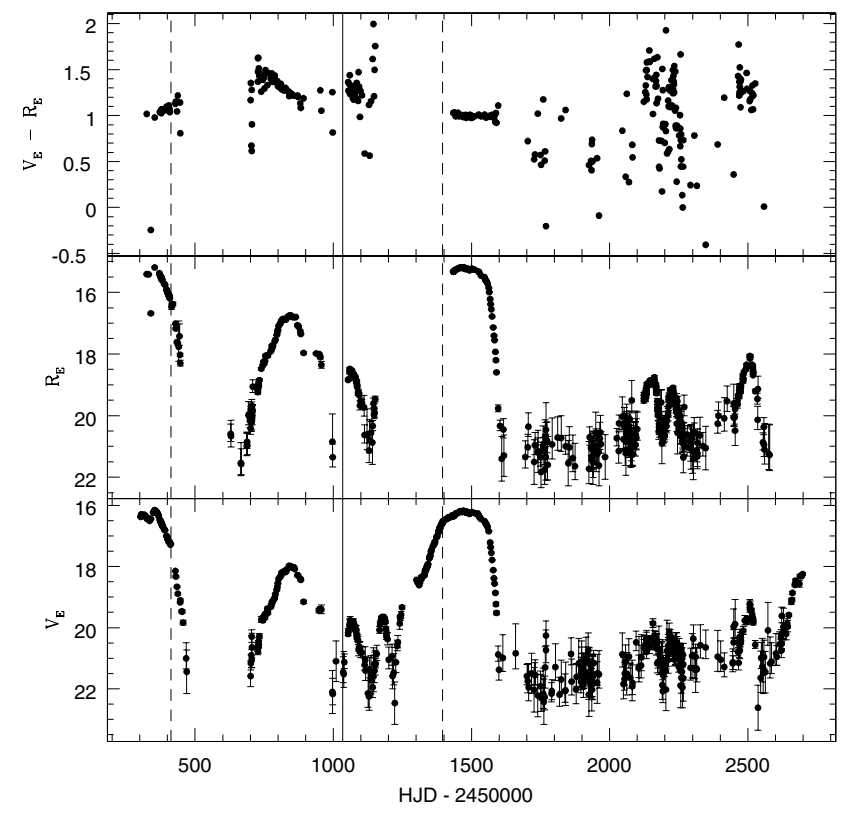

Fig. 1. EROS light curves of RAW 21. The vertical solid line indicates the epoch of the 2MASS observation while dashed lines are DENIS observations.

\subsection{RAW 21}

From its carbon-rich spectra with strong $\mathrm{C}_{2}$ bands but very weak CN bands (Morgan et al. 2003), this star was proposed as the first RCB candidate ever detected in the SMC. But its variability data were too sparse until now to definitively conclude on its nature. Thanks to the EROS 2 light curve revealing huge brightness variations, we can claim that RAW 21 is indeed the first RCB discovered in the SMC.

Figure 6 shows that a standard extinction does not explain what is observed during the phases of luminosity changes. In addition, a drastic change in $J$ and $K$ magnitudes is observed when comparing the values from 2MASS and DENIS given in Table 2. This reflects the different epochs of the 2MASS and DENIS observations. The optical EROS 2 data collected at these epochs also reveal that RAW 21 was brighter when observed by DENIS. Furthermore, Morgan et al. (2003) assumed that this RCB was much fainter in June 2000 than in January 2000 since they failed to collect a spectrum in June. EROS 2 data also confirm such a variation. 


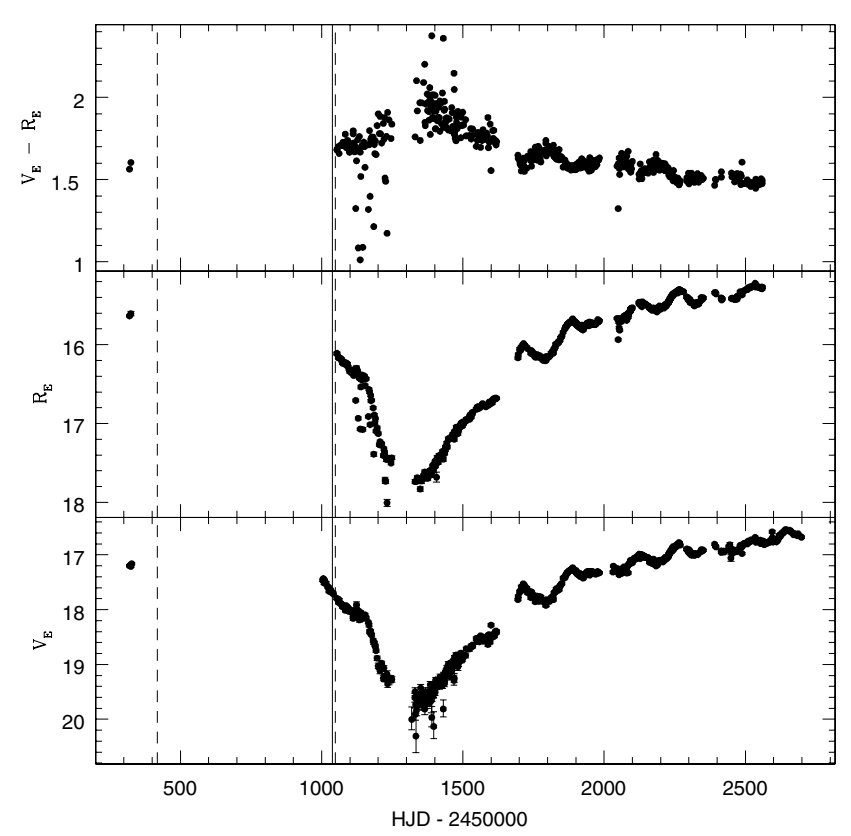

Fig. 2. EROS light curves of RAW 233. The vertical solid line indicates the epoch of the 2MASS observation while dashed lines are DENIS observations.

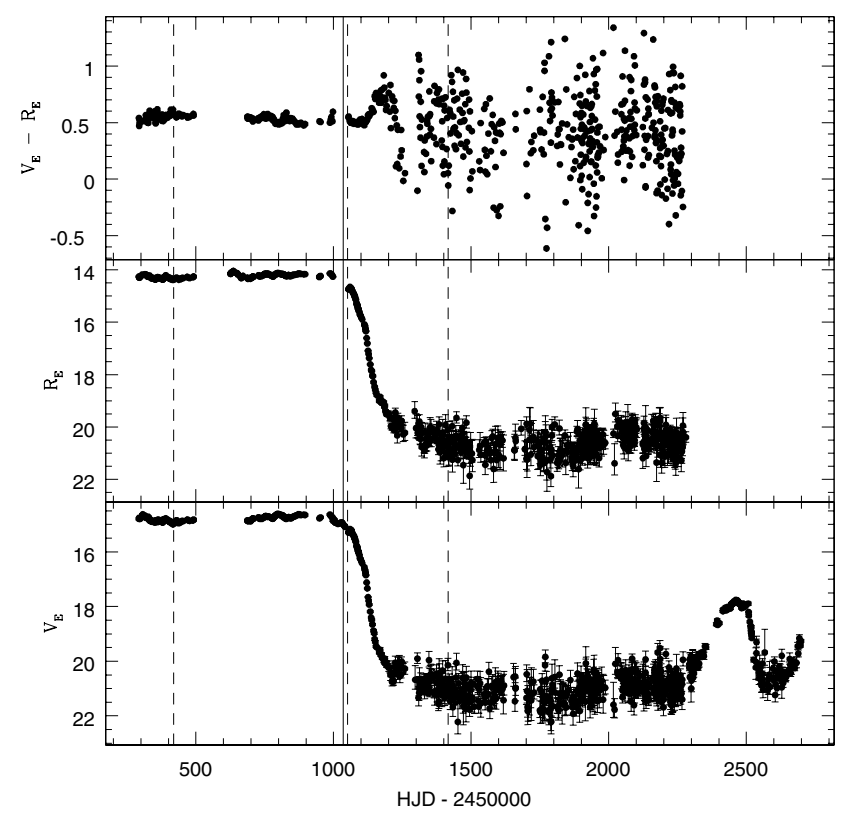

Fig. 3. EROS light curves of RAW 476. The vertical solid line indicates the epoch of the 2MASS observation while dashed lines are DENIS observations.

Finally, the temporal evolution of $J-K$ suggests that this object has experienced a major episode of local extinction which is also observed in the optical domain as shown in Fig. 1. This is consistent with the classical interpretation for the RCB brightness declines assuming that they are caused by successive obscurations by dust clouds.

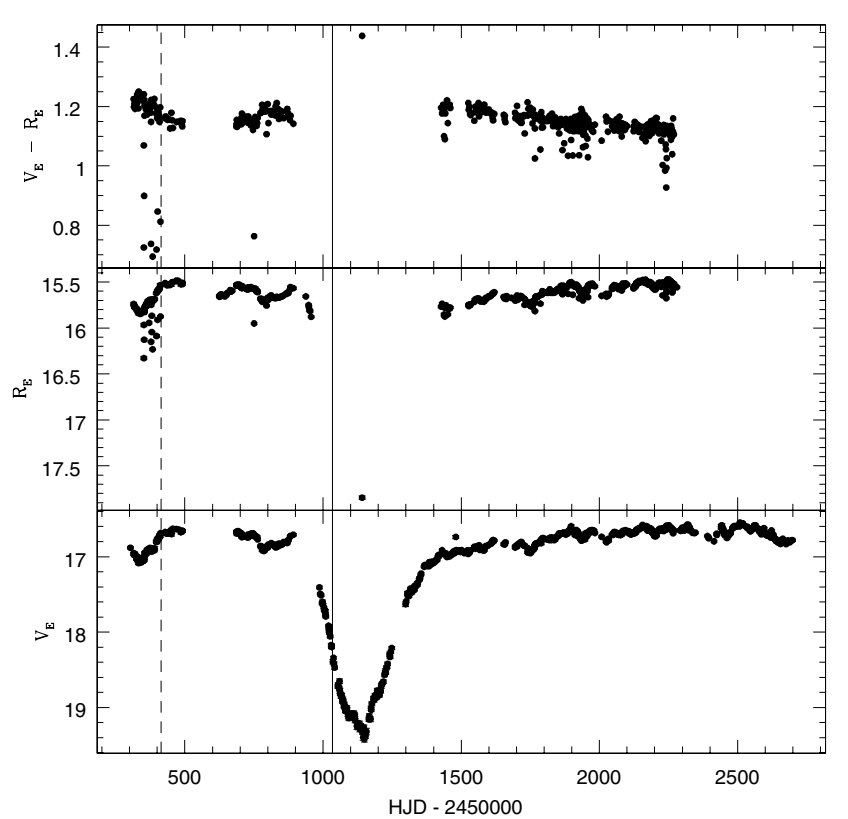

Fig. 4. EROS light curves of [MH95] 431. The vertical solid line indicates the epoch of the 2MASS observation while the dashed line is the DENIS observation.

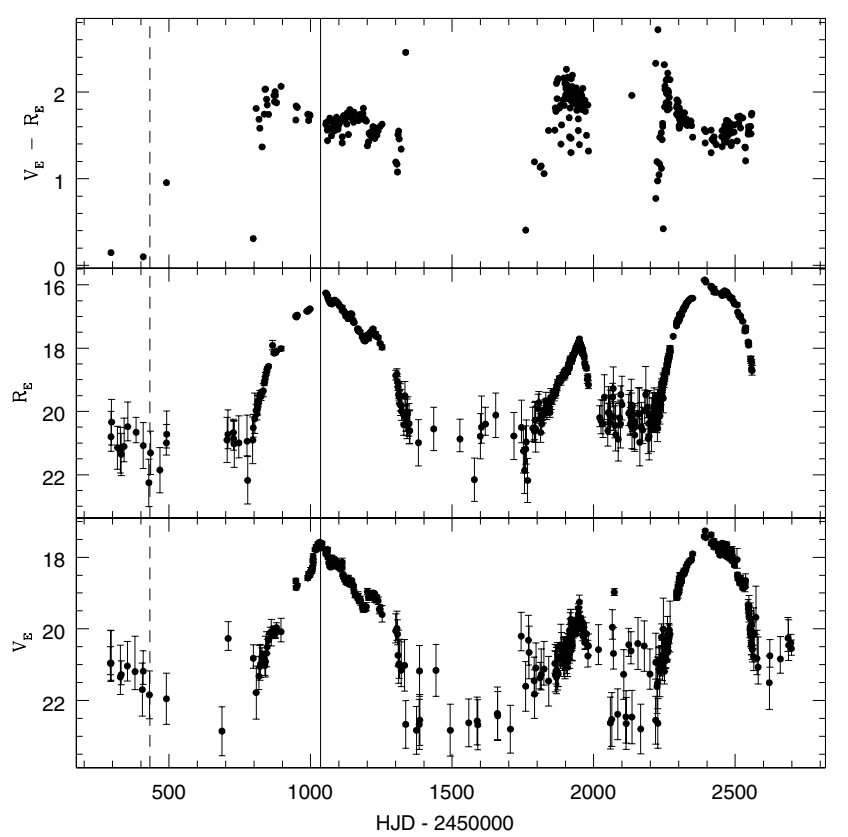

Fig. 5. EROS light curves of sm0067m28134b. The vertical solid line indicates the epoch of the 2MASS observation while the dashed line is the DENIS observation.

\subsection{RAW 233}

The EROS 2 lightcurve shown in Fig. 2 is a very good example of RCB brightness variations with (i) a large decline of about 2-3 mag, (ii) a larger decline at shorter wavelengths compatible with dust obscuration, and (iii) regular or semiregular pulsations around maximum light with amplitudes of a few tenths of magnitude and pseudo-periods of a few tens of days. The MACHO light curves collected between JD 2448800 and JD 2451400 exhibit an earlier episode of 

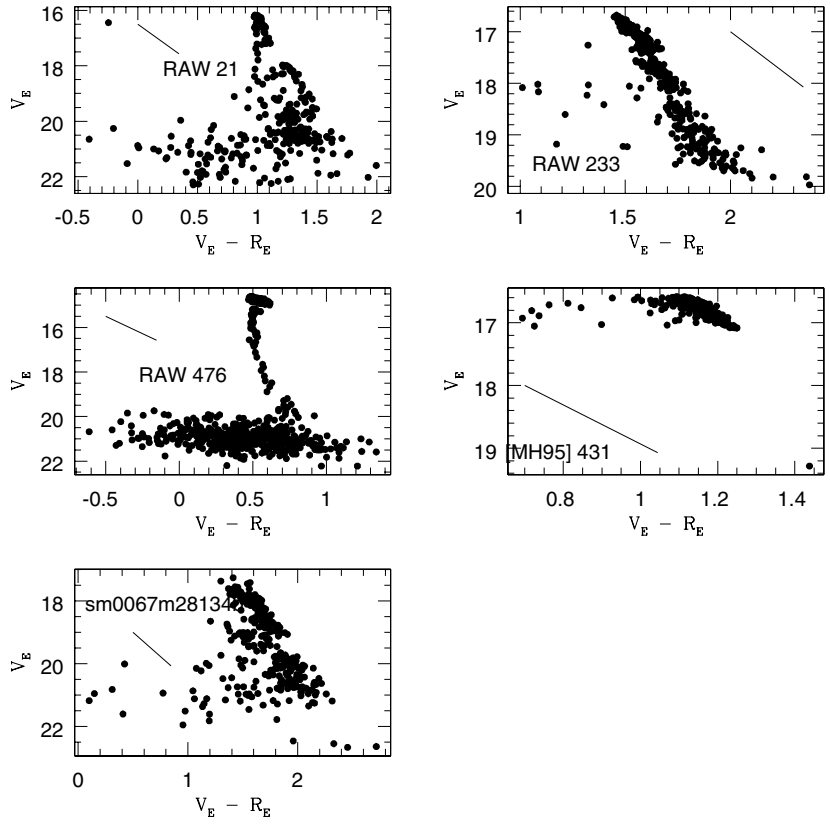

Fig. 6. Colour-magnitude diagram of the RCB candidates. The solid lines give the direction of the reddening vectors whose determination is discussed in the text.

slow magnitude decrease and increase with superimposed similar low amplitude variations as those observed in Fig. 2.

The colour diagram in Fig. 6 for this star shows a decrease which is steeper than the extinction vector. The almost symmetrical brightness decrease and increase and the position of this object in Fig. 7 are comparable with that of DY Per stars (cooler and fainter Galactic RCB with slower symmetrical declines) indicated by Alcock et al. (2001) and close to the line giving the colour of the SMC carbon stars by Westerlund et al. (1991).

From our photometric data and its already known carbonrich nature, we therefore propose that RAW 233 is the first $\mathrm{RCB}$ of the DY Per-type detected in the SMC. Its cooler effective temperature must be confirmed spectroscopically.

\subsection{RAW 476}

The evolution of this carbon-rich object in Fig. 3 is the most spectacular with a decline of almost 7 mag after a long episode of relative stability. We also point out that very low-amplitude pulsations are detected at maximum light and the decline is deeper at shorter wavelengths. The extinction in $V_{\mathrm{E}}$ in Fig. 6 is practically colour-independent. The Combined General Catalog of Variable Stars (Kholopov et al. 1998) reports for this object a magnitude at maximum brightness of 15.4 and an irregular type of variability. Its RCB nature might thus be definitively established if this star has recovered to this maximum brightness after the EROS 2 observations and if its spectrum exhibits strong $\mathrm{C}_{2}$ and weak $\mathrm{CN}$ bands.

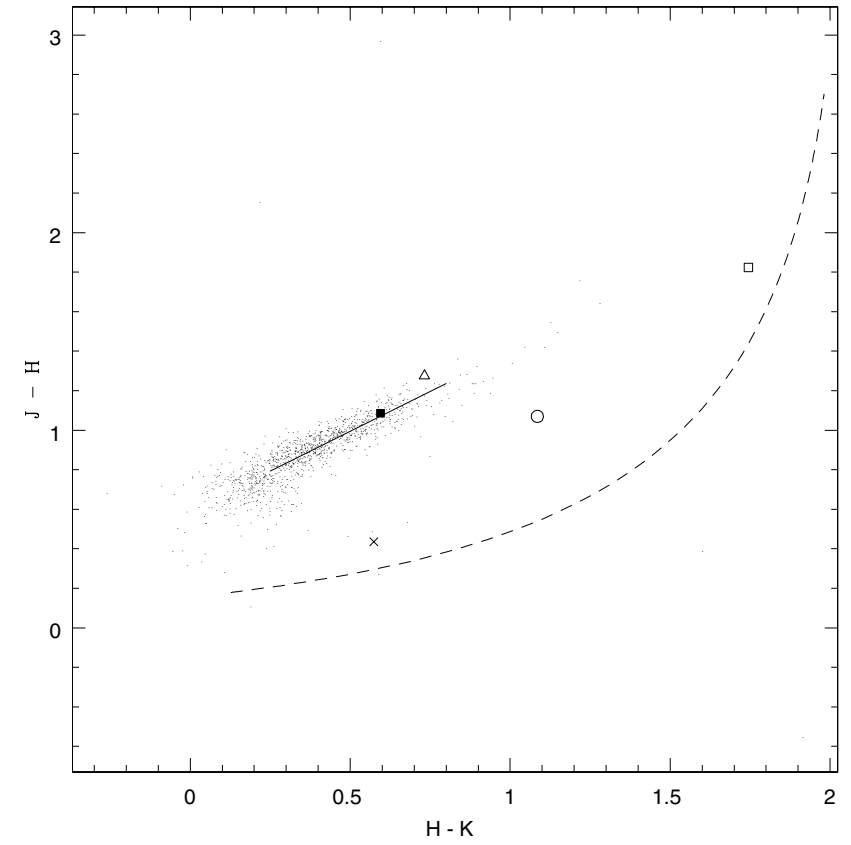

Fig. 7. $J-H$ versus $H-K$ two-colour diagram. Solid dots: 2MASS data collected through VizieR for the 1185 carbon stars of the catalog by Morgan \& Hatzidimitriou (1995); open square: RAW 21; open triangle: RAW 233; cross: RAW 476; filled square: [MH95] 431; open circle: sm0067m28134b. The solid line represents the colours of SMC carbon stars as parameterised by Westerlund et al. (1991). The dashed line is the locus of a combination of $5500 \mathrm{~K}$ (the "star") and $1000 \mathrm{~K}$ (the "shell") blackbodies in various proportions ranging from all "star" (lower end) to all "shell" (upper end), as calculated by Feast (1997).

\section{4. [MH95] 431}

The carbon star [MH95] 431 is another very good RCB candidate. It exhibits in Fig. 4 low amplitude and colour-dependent magnitude changes at maximum light and a huge decline in $V_{\mathrm{E}}$. However, the lack of data in $R_{\mathrm{E}}$ during the main decline rules out any definitive conclusion on a reddening at this epoch. Notice however that the points at JD 2451130 are real and are compatible with the dust obscuration scenario. Here again low amplitude variations are observed and the two-colour diagram of Fig. 7 shows an almost perfect agreement with the colour of SMC carbon stars of Westerlund et al. (1991). Again, this object is located in the same region of the diagram as the DY Per stars of Alcock et al. (2001). Its almost symmetrical decline is indeed compatible with a possible DY Per nature; its cooler temperature should be checked spectroscopically.

\section{5. $s m 0067 m 28134 b$}

This star is the only one which is not included in a published catalog of carbon stars. This could be explained by the fact that it is very faint on the EROS template image as can be seen on the finding chart of Fig. 8. On the other hand there is at this location a quite bright 2MASS source (see Table 2). In the same way the GSC 2.2 catalog available through VizieR yields a source with a $F$ photographic magnitude of 17.04 while visible magnitudes are not determined. This strongly suggests the 
Table 2. 2MASS and unpublished DENIS infrared data for the EROS 2 SMC RCB candidates.

\begin{tabular}{llllllll}
\hline \hline Identifier & JD Epoch & $J_{2 \text { MASS }}$ & $H_{2 \text { MASS }}$ & $K_{\text {2MASS }}$ & $I_{\text {DENIS }}$ & $J_{\text {DENIS }}$ & $K_{\text {DENIS }}$ \\
\hline RAW 21 & 2451033.9 & 17.06 & 15.23 & 13.49 & & & \\
& 2450412.6 & & & & 15.72 & 14.65 & 12.43 \\
& 2451394.9 & & & & 14.89 & 13.66 & 11.87 \\
RAW 233 & 2451034.7 & 13.31 & 12.03 & 11.30 & & & \\
& 2450040.6 & & & & 15.29 & 13.43 & 11.30 \\
& 2450418.6 & & & & 14.91 & 13.13 & 11.05 \\
& 2451048.8 & & & & 15.40 & 13.37 & 11.27 \\
RAW 476 & 2451034.7 & 13.12 & 12.69 & 12.11 & & & \\
& 2450418.6 & & & & 13.70 & 13.11 & 11.98 \\
& 2451035.9 & & & & 13.76 & 13.11 & 12.02 \\
& 2451050.8 & & & & 13.99 & 13.32 & 12.15 \\
& 2451416.8 & & & & nd & nd & 13.16 \\
& 2451033.9 & 14.63 & 13.54 & 12.95 & & & \\
& 2450413.6 & & & & 14.73 & 13.33 & 12.06 \\
& 2450414.6 & & & & 14.82 & 13.30 & 11.78 \\
& 2451034.8 & 13.88 & 12.81 & 11.73 & & & \\
\hline Sm0067m28134b 431 & 2450432.6 & & & & nd & nd & 13.71 \\
\hline
\end{tabular}

nd $=$ not detected.

presence of a star embedded in a screening dust shell and visible at irregular intervals. The MACHO light curve is available in one colour only; it displays an earlier and even larger variation of about 6 mag between the epochs JD 2449300 and JD 2450000. If its RCB nature is confirmed spectroscopically, this object has undergone a large number of obscurations by dust clouds revealing an interesting phase of dust ejections.

\subsection{Comparison with $L M C$ objects}

The steepest luminosity decrease observed for our 5 candidates are of order $0.01 \mathrm{mag} / \mathrm{day}$ for the 2 possible DY Per, and $0.06-0.12 \mathrm{mag} / \mathrm{day}$ for the other three; these values are very similar to those given by Alcock et al. (2001).

Figure 9 shows the 2MASS $K$ versus $H-K$ colour-magnitude diagram of our RCB candidates (left panel) compared to the RCB stars found in the LMC (right panel). For 3 LMC objects out of 17, no obvious 2MASS counterpart was found and they are omitted in the figure. The solid black dots are stars chosen as follows: for each RCB candidate a query was made on the 2MASS catalog through VizieR to extract 1000 sources within a radius of $10^{\prime}$ around the object and with a $J H K$ photometric quality flag equal to AAA. Finally, the red dots represent either the catalog of Morgan \& Hatzidimitriou (1995) (for SMC) or that of Kontizas et al. (2001) (for LMC).

From this figure, it appears that both the SMC \& LMC carbon star populations show similar patterns and that our SMC RCB candidates are located in similar regions

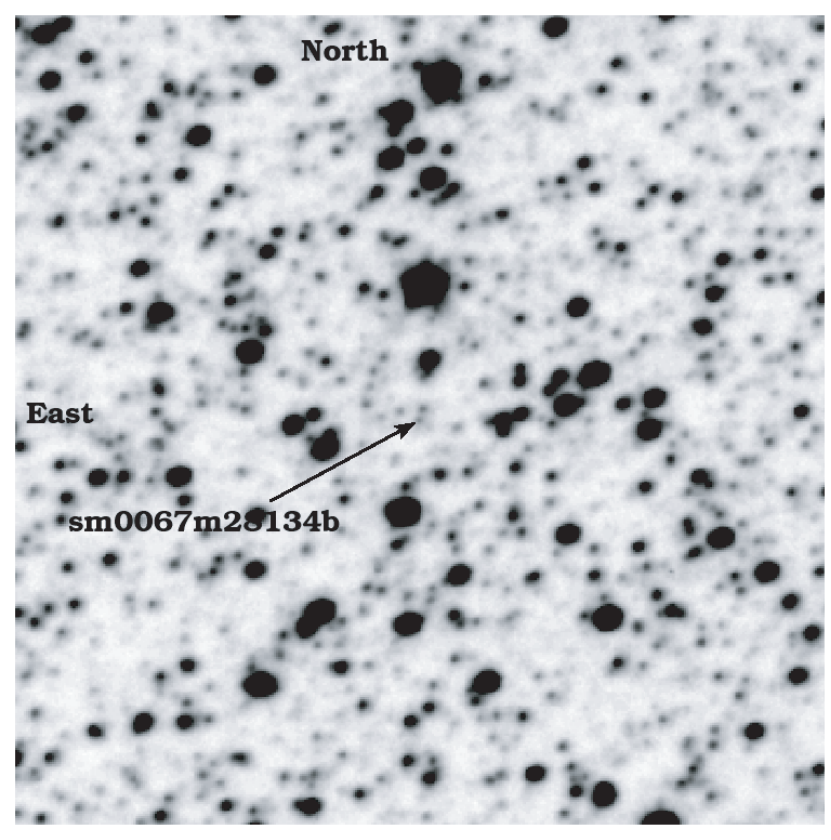

Fig. 8. Finding chart of sm0067m $28134 \mathrm{~b}$ which is indicated by the arrow. The size of the frame is $168^{\prime \prime} \times 168^{\prime \prime}$. North is up, East is left.

with respect to these carbon stars. Notice that the four LMC DY Per stars proposed by Alcock et al. (2001) are well separated from the other objects (four upper crosses in the right panel of Fig. 9) while no such difference is seen on the SMC part of the figure. This indicates that the DY Per-like position of RAW 233 and [MH95] 431 in Fig. 7 is not confirmed 


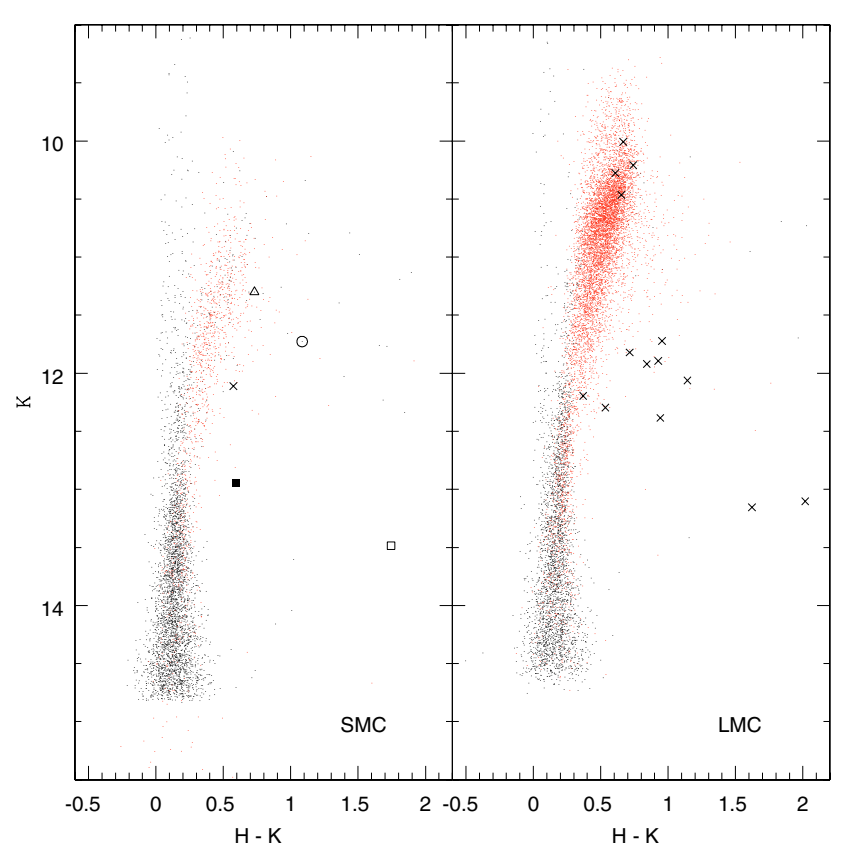

Fig. 9. $K$ versus $H-K$ colour-magnitude diagram. Left panel: SMC; the symbols for our 5 RCB candidates are the same as in Fig. 7; black solid dots are discussed in the text; red solid dots are the carbon stars of the catalog by Morgan \& Hatzidimitriou (1995). Right panel: LMC; crosses represent RCB stars given by Alcock et al. (2001) in their Table 1 for which 2MASS data exist; black solid dots are discussed in the text; red solid dots are 7609 carbon stars extracted from the catalog of Kontizas et al. (2001).

in Fig. 9. Clearly spectroscopic measurements are necessary to disentangle this question.

Finally, we have estimated the absolute visual magnitude at maximum light $\left(M_{V}\right.$ in the Johnson system) of the RCB candidates in the SMC. They are presented in Table 3 together with their colour at maximum light. For that purpose, we assumed a distance modulus of 18.9 (Harries et al. 2003) and $A_{V}=0.123$ (NED), constant over the whole SMC field covered by EROS 2 . We neglected the circumstellar extinction around each RCB. The standard $V$ and $I$ magnitudes are estimated within a $10 \%$ accuracy for each field and CCD by matching the EROS catalogue of stars with those from DENIS and OGLE$^{2}$ (Udalski et al. 1998).

It should be noted that, for the first four stars in Table 3, we are confident that the quoted magnitudes and colours correspond to the maximum luminosity. For sm0067m28134b in contrast, we have evidence from the MACHO data that the maximum luminosity in Fig. 5 is about 2 mag fainter than the actual maximum.

RAW 21 and RAW 476 have absolute luminosities compatible with the faintest $\left(M_{V} \sim-2.5\right)$ and the brightest $\left(M_{V} \sim\right.$ $-5) \mathrm{RCB}$ of the LMC respectively. It can also be seen that the faintest RCB candidates are the reddest as already noticed for LMC RCB (see Alcock et al. 2001). The two DY Per candidates of the SMC (RAW 233 and [MH95] 431) are the coolest SMC candidates and have an absolute luminosity slightly

\footnotetext{
2 Available at the URL

http://sirius. astrouw.edu.pl/ ogle/ogle2/smc_maps.html
}

Table 3. Estimated absolute luminosity and colour at maximum light of the RCB candidates in the SMC.

\begin{tabular}{lcc}
\hline \hline RCB candidate & $M_{\mathrm{V}}$ & $(V-I)$ \\
\hline RAW 21 & -2.5 & 1.9 \\
RAW 233 & -2.1 & 2.1 \\
RAW 476 & -4.5 & 1.0 \\
[MH95] 431 & -2.1 & 2.0 \\
sm0067m28134b & -1.3 & 2.4 \\
\hline
\end{tabular}

fainter than, but still compatible with, that of LMC DY Per stars $\left(M_{V} \sim-2.5\right)$. Finally, conclusions on sm0067m28134b are more uncertain as EROS 2 did not observe this star at maximum light, while MACHO likely observed it but only in one passband.

To summarize, we find that all the RCB candidates in the SMC follow the classical relationship between absolute magnitude and effective temperature for the RCB variables and have absolute luminosities close to those found in the LMC.

\section{Summary}

A search has been performed for RCB candidates in the SMC among 4.2 millions light curves of the EROS 2 database. After applying various filters described in Sect. 3, five objects have been identified as possible stars undergoing the RCB phase of evolution (large declines, infrared excess at minimum light and semi-regular variations at maximum). Four of them being previously known as carbon stars, their RCB nature is reinforced.

One of them, RAW 21, being already known as exhibiting strong $\mathrm{C}_{2}$ bands but very weak $\mathrm{CN}$-bands, is therefore the first confirmed RCB found in the SMC with an absolute luminosity close to that of the faintest known RCB in the LMC. On the other hand, RAW 476 is almost as bright as the brightest known RCB.

From their position in the $J-H$ versus $H-K$ two-colour diagram, their absolute luminosity and their symmetrical decline in the lightcurve, the RCB type of the stars RAW 233 and [MH95] 431 is possibly DY Per-like although no such evidence appears on the $K$ versus $H-K$ colour-magnitude diagram.

With the data for RAW 21, RAW 476 and $s m 0067 \mathrm{~m} 28134 \mathrm{~b}$, a linear correlation is observed in the $J-H$ versus $H-K$ two-colour diagram. The SMC candidates have similar infrared colours with respect to carbon stars as do LMC stars.

Little can be said about the abundance of RCB stars with only five candidates. Based on their study of galactic RCB stars, Alcock et al. (2001) suggested that only $\sim 10^{-6}$ of all stars are RCB stars. We find that in the SMC, $0.7-1.7 \times$ $10^{-6}(1 \sigma)$ of all stars display photometric features characteristic of RCB stars over the course of 6.5 years. (Taking into account our estimated detection efficiency, the actual fraction of RCB stars may be up to twice higher.) Thus, there is no significant detection of any environmental, historical, or metallicity dependence on the fraction of RCB stars. 
Finally, we suggest that spectroscopic studies checking the relative strength of the $\mathrm{C}_{2}$ and $\mathrm{CN}$ bands be carried out to definitively confirm the RCB nature of RAW 233, RAW 476, [MH95] 431 and sm0067m28134b.

Acknowledgements. We thank the anonymous referee for his/her useful comments. This research has made use of VizieR and the Simbad database, operated at CDS, Strasbourg, France. This publication makes use of data products from the Two Micron All Sky Survey, which is a joint project of the University of Massachusetts and the Infrared Processing and Analysis Center/California Institute of Technology, funded by the National Aeronautics and Space Administration and the National Science Foundation. The unpublished DENIS data have been kindly provided by Gary Mamon. The DENIS project has been partly funded by the SCIENCE and the HCM plans of the European Commission under grants CT920791 and CT940627. It is supported by INSU, MEN and CNRS in France, by the State of Baden-Württemberg in Germany, by DGICYT in Spain, by CNR in Italy, by FFwFBWF in Austria, by FAPESP in Brazil, by OTKA grants F-4239 and F-013990 in Hungary, and by the ESO C\&EE grant A-04-046.

\section{References}

Afonso, C., Albert, J. N., Alard, C., et al. 2003, A\&A, 404, 145 Alcock, C., Allsman, R. A., Alves, D. R., et al. 1996, ApJ, 470, 583
Alcock, C., Allsman, R. A., Alves, D. R., et al. 2001, ApJ, 554, 298 Ansari, R. 1996, Vis. Astron., 40, 519

Cardelli, J. A., Clayton, G. C., \& Mathis, J. S. 1989, ApJ, 345, 245

Clayton, G. C. 1996, PASP, 108, 225

Derue, F., Afonso, C., Alard, C., et al. 2001, A\&A, 373, 126

Derue, F., Marquette, J.-B., Lupone, S., et al. 2002, A\&A, 389, 149

Feast, M. W. 1997, MNRAS, 285, 339

Harries, T. J., Hilditch, R. W., \& Howarth, I. D. 2003, MNRAS, 339, 157

Kholopov, P. N., Samus, N. N., Frolov, M. S., et al. 1998, in Combined general catalogue of variable stars, $4.1 \mathrm{ed}, \mathrm{II} / 214 \mathrm{~A}$, available through VizieR

Kontizas, E., Dapergolas, A., Morgan, D. H., \& Kontizas, M. 2001, A\&A, 369, 932

Lasserre, T., Afonso, C., Albert, J. N., et al. 2000, A\&A, 355, L39

Morgan, D. H., \& Hatzidimitriou, D. 1995, A\&AS, 113, 539

Morgan, D. H., Hatzidimitriou, D., Cannon, R. D., \& Croke, B. F. W. 2003, MNRAS, 344, 325

Palanque-Delabrouille, N., Afonso, C., Albert, J. N., et al. 1998, A\&A, 332,1

Rebeirot, E., Azzopardi, M., \& Westerlund, B. E. 1993, A\&AS, 97, 603

Udalski, A., Szymanski, M., Kubiak, M., et al. 1998, Acta Astron., 48, 147

Westerlund, B. E., Azzopardi, M., Rebeirot, E., \& Breysacher, J. 1991, A\&AS, 91, 425 